Recibido: 24/05/2021 --- Aceptado: 06/09/2021 --- Publicado: 27/09/2021

\title{
EL FACT-CHECKER EN ESPAÑOL ALREDEDOR DEL MUNDO: PERFIL, SIMILITUDES Y DIFERENCIAS ENTRE VERIFICADORES HISPANOHABLANTES
}

\section{SPANISH-SPEAKING FACT CHECKERS AROUND THE WORLD: PROFILES, SIMILARITIES AND DIFFERENCES AMONG FACT CHECKING PROFESSIONALS}

88 $R^{a}$ Esperanza Herrero1: Universidad de Murcia. España. mariaesperanza.herrero@um.es

Susana Herrera Damas²: Universidad Carlos III de Madrid. España. dherrera@hum.uc3m.es

Investigación financiada por el Ministerio de Educación y Formación Profesional de España mediante Beca de Colaboración 2019/20 con el Departamento de Comunicación de la Universidad Carlos III de Madrid

Cómo citar el artículo:

Herrero, E. y Herrera Damas, S. (2021). El fact-checker en español alrededor del mundo: Perfil, similitudes y diferencias entre verificadores hispanohablantes. Revista de Comunicación de la SEECI, 54, 49-77. http://doi.org/10.15198/seeci.2021.54.e725

\section{RESUMEN}

El del fact-checker es un rol profesional cada vez más relevante en el control de los procesos informativos contemporáneos. Sin embargo, su perfil ha sido poco abordado a nivel internacional, y todavía menos en el contexto hispanohablante. El fact-checking es un fenómeno que se sitúa al centro de los procesos informativos contemporáneos alrededor del mundo, convirtiéndose en un movimiento periodístico global en crecimiento desde su consolidación en el panorama anglosajón. Particularmente, la verificación de informaciones en español está viviendo un crecimiento exponencial en los últimos años con un aumento de más del $500 \%$ del número de plataformas desde 2018. Conocer el perfil de quienes desarrollan la verificación de informaciones en países de habla hispana es esencial para estudiar en profundidad el fenómeno dentro

\footnotetext{
${ }^{1}$ Esperanza Herrero: Investigadora predoctoral FPU/MECD en el Departamento de Comunicación de la Facultad de Comunicación y Documentación de la Universidad de Murcia (España). mariaesperanza.herrero@um.es

${ }^{2}$ Susana Herrera Damas: Profesora titular de Periodismo en el Departamento de Comunicación de la Universidad Carlos III de Madrid (España) donde dirige el programa de doctorado en Investigación en Medios de Comunicación.

dherrera@hum.uc3m.es
} 
Herrero, E. y Herrera Damas, S. El fact-checker en español alrededor del mundo: Perfil, similitudes y diferencias entre verificadores hispanohablantes

de un contexto de convergencia como el hispanoamericano. Para ello, se lleva a cabo una encuesta $(n=52)$ entre verificadores hispanohablantes con el objetivo de conocer su percepción sobre la vinculación de esta actividad con el periodismo, así como el grado de colaboración que existe entre las distintas iniciativas y las similitudes y diferencias entre los fact-checkers de España y América Latina. En un segundo momento, se enriquecen los resultados con la realización de 13 entrevistas en profundidad a profesionales y estudiosos del fenómeno.

PALABRAS CLAVE: Verificación - verificador - periodismo - plataformas desinformación - innovación - verdad - España - América Latina

\section{ABSTRACT}

Professional fact-checkers are becoming essential for the control of information and disinformation flows. However, their profile has been scarcely studied both internationally and particularly, in the Spanish-speaking context. Fact-checking is now a global journalism movement, after being consolidated in Anglo-Saxon countries, and has become a key for understanding current mediated informative and communicative processes that take place in democracies around the world. Spanish-speaking factchecking is growing exponentially in recent years, with an increase of more than $500 \%$ in the number of active platforms since 2018. Studying the profile of Spanish-speaking fact-checkers is crucial to understand the phenomenon in a context of constant convergence such as the Hispanic American. A survey $(n=52)$ was conducted among Spanish-speaking fact-checkers to analyze their perception of fact-checking's link to journalism, the level of collaboration among platforms, as well as similitudes and differences between Spanish and Latin American professionals. After that, results are enriched through 13 in-depth interviews to fact-checking professionals and academic experts.

KEY WORDS: Fact-checking - fact-checker - journalism - platforms - disinformation - innovation - truth - Spain - Latin America

\section{O FACT-CHECKER EM ESPANHOL AO REDOR DO MUNDO: PERFIL, SEMELHANÇAS E DIFERENÇAS ENTRE VERIFICADORES DE FALA EM ESPANHOL}

\section{RESUMO:}

O fact-checker é um papel profissional cada vez mais relevante no controle dos processos de informação contemporâneos. No entanto, seu perfil tem sido pouco abordado internacionalmente, e menos ainda no contexto da língua espanhola. O fact-checking é um fenômeno que está no centro dos processos noticiosos contemporâneos em todo o mundo, tornando-se um movimento jornalístico global crescente desde sua consolidação no cenário anglo-saxão. Em particular, ao fact-checking em espanhol está experimentando um crescimento exponencial nos últimos anos com um aumento de mais de $500 \%$ no número de plataformas desde 2018. Conhecer o perfil de quem realiza a verificação de informações em países de língua espanhola é essencial para estudar em profundidade o fenômeno dentro de um contexto de convergência como o do hispano-americano. Para isto, foi realizado um

Revista de Comunicación de la SEECI. 2021, nº 54, 49-77 
Herrero, E. y Herrera Damas, S. El fact-checker en español alrededor del mundo: Perfil, similitudes y diferencias entre verificadores hispanohablantes

inquerito ( $n=52)$ a verificadores de língua espanhola para conhecer a sua percepção sobre a ligação desta actividade com o jornalismo, bem como o grau de colaboração existente entre as diferentes iniciativas e as semelhanças e diferenças entre fact-checkers na Espanha e na América Latina. Em um segundo momento, os resultados são enriquecidos pela realização de 13 entrevistas em profundidade com profissionais e estudiosos do fenômeno.

PALAVRAS-CHAVE: Verificação - verificador - jornalismo - plataformas - desinformação inovação - verdade - Espanha - América Latina.

\section{INTRODUCCIÓN}

El fact-checker se ha convertido en los últimos años en un profesional clave en los procesos informativos, comunicativos y democráticos que se producen en la sociedad contemporánea. Sin embargo, la investigación sobre verificación de informaciones ha puesto mayoritariamente el foco en los análisis de contenido de los bulos o en las metodologías de fact-checking y no tanto en el rol de los profesionales que se dedican al control de veracidad del discurso público. En general, el fact-checking es una práctica de control de veracidad del discurso público cada vez más consolidada que, aunque nacida en el marco periodístico (Graves y Glaisyer, 2012), vienen desempeñando plataformas de distinta índole, tanto mediáticas como empresariales. Sus raíces periodísticas han llevado a Bill Adair, profesor de la Universidad de Duke y fundador de la plataforma PolitiFact, a hablar del fact-checking como "la variante más importante del periodismo en la era digital" (Adair, 2019) y al profesor de la Universidad de Wisconsin-Madison, Lucas Graves (2016, p. 2), a referirse al factchecking como "un raro ejemplo de un movimiento periodístico genuinamente transnacional".

La crisis global desencadenada por la pandemia por COVID-19 tuvo un especial impacto en el aumento de la circulación de desinformación, llevando a la Organización Mundial de la Salud (OMS) a hablar de una "infodemia" y a llamar a la acción a distintos actores internacionales para promover el acceso a informaciones veraces (OMS, 11 de diciembre de 2020). Pese a ser un movimiento global, el fact-checking venía abordando la desinformación desde la particularidad de los contextos locales. Sin embargo, un fenómeno verdaderamente global como la pandemia ha favorecido una interconexión entre fact-checkers que no conoce fronteras. Como consecuencia del aumento de la desinformación, durante el año 2020, el número de plataformas de verificación creció exponencialmente a nivel internacional según un informe del Reuters Institute (Brennen et al., 2020) y, de manera específica, también lo hizo en el mundo hispanohablante (Duke Reporters' Lab, 2020).

Como venimos diciendo, las acciones de control de la desinformación son desarrolladas tanto por medios de comunicación institucionalizados (Graves y Glaisyer, 2012) como por plataformas digitales independientes o iniciativas activistas. Esto ha generado un debate en torno a la vinculación de las prácticas de verificación con el periodismo tradicional. Graves y Cherubini (2016, p. 3) se aproximan a los factcheckers distinguiendo dos categorías: lo que dan en llamar "el modelo de redacción", 
Herrero, E. y Herrera Damas, S. El fact-checker en español alrededor del mundo: Perfil, similitudes y diferencias entre verificadores hispanohablantes

vinculado a los medios de comunicación, y el "modelo ONG", con una vocación más activista.

Sin embargo, en los últimos años, son también cada vez más las redes sociales y las empresas digitales -como Twitter, Facebook, Google o YouTube- las que se han involucrado en el control de los flujos de desinformación, desdibujando así, en cierto modo, la conexión entre las prácticas de verificación y la labor periodística tradicional. Esta irrupción ha abierto la puerta a nuevas propuestas metodológicas de control informativo y a la automatización de los procesos de fact-checking (Hassan et al., 2017; Cazalens et al., 2018; Graves, 2018), generando un debate en torno a la oportunidad del empleo de la inteligencia artificial, la elevada complejidad de los procesos informativos sociales y la subjetividad de la realidad social. Graves (2018, p. 1) se refiere a la automatización de la verificación como una manera de "buscar soluciones tecnológicas a lo que es visto como un problema tecnológico", una manera de simplificar la complejidad de la realidad informativa y dejar a un lado la necesidad de filtros subjetivos.

Si bien es cierto que la necesidad de competencias relativas a la inteligencia artificial y las herramientas digitales están convirtiendo al del profesional del fact-checking en un perfil cada vez más especializado (Vizoso et al., 2018), también encontramos que este viraje hacia la automatización sirve para reivindicar la relevancia de los factcheckers humanos, capaces de trabajar en el control de la desinformación en una realidad democrática que frecuentemente, como plantea Uscinski (2015), no es verificable, o no se puede dividir en blancos y negros.

Como hemos visto, las perspectivas que tienen sobre su trabajo los profesionales de la verificación han sido poco abordadas por la literatura académica a nivel internacional (IFCN, 2016; Graves y Cherubini, 2016; Ginsberg y Gori, 2021), e incluso en menor medida en el contexto hispanohablante (Moreno Gil et al., 2021). Pese a su naturaleza periodística, Cazalens et al. (2018, p. 566) distinguen al fact-checking de los procesos tradicionales del periodismo hablando de "verificación de informaciones a priori" -la realizada por los periodistas para controlar la veracidad de las informaciones propias antes de publicarlas- y de "verificación de informaciones a posteriori", o el fact-checking per se, que tiene como objetivo controlar la veracidad de declaraciones e informaciones ajenas.

En efecto, el "fact-checking a priorl" del que hablan Cazalens et al. (2018) es una práctica esencial al periodismo desde su nacimiento (Kovach y Rosenstiel, 2007; Ciampaglia et al., 2015), lo que explica que haya sido, precisamente, en la esfera periodística en la que han surgido y en la que se mueven la mayor parte de iniciativas de fact-checking a posteriori (Graves y Glaisyer, 2012). Sin embargo, la distinción de Cazalens et al. (2018) nos parece relevante para entender la manera en la que, pese a su estrecha vinculación con el periodismo, el fact-checking es un fenómeno claramente diferenciado que, además, se esta extendiendo fuera de los límites de los medios de comunicación y las plataformas periodísticas. 
Herrero, E. y Herrera Damas, S. El fact-checker en español alrededor del mundo: Perfil, similitudes y diferencias entre verificadores hispanohablantes

El fact-checking a posteriori va más allá del periodismo tradicional y aparece allí donde los métodos de verificación de los medios de comunicación no pueden hacer frente a la sobreinformación (Ciampaglia et al., 2015). En este ecosistema de sobreabundancia informativa, la verificación se considera "la mejor herramienta" para contrarrestar el poder de las noticias falsas (Amorós, 2018, p. 154), y evitar el riesgo que su difusión descontrolada supone para los sistemas democráticos (Rose, 2017; Del Fresno, 2018). En esta línea, entendemos que las noticias falsas no son solo un problema mediático sino, como indica Persily (2017), también un fenómeno social, de manera que la respuesta periodística a la desinformación no sería exclusivamente una respuesta a un problema de falta de credibilidad de las instituciones mediáticas (Algan et al., 2017, Persily, 2017), sino a un problema, en esencia, democrático (Rose, 2017; Del Fresno, 2018).

La desinformación, en efecto, no tiene ya exclusivamente consecuencias mediáticas y políticas, sino también consecuencias para la salud pública, como se ha comprobado a través de la infodemia de la que hablaba la OMS. La $B B C$ llegó a titular "Hundreds dead' because of COVID-19 misinformation" (cientos de muertos por la desinformación sobre la COVID-19), en un artículo que reflexionaba sobre el impacto de las noticias falsas que circularon durante los primeros meses de la pandemia (Coleman, 2020); una cuestión tratada también por revistas de divulgación científica como Scientific American, que denunciaba: "COVID misinformation is killing people" (la desinformación sobre la COVID está matando gente) (Scientific American, 11 de octubre de 2020). La desinformación sobre salud, ciencia y vacunaciones ha sido considerada un riesgo para la ciudadanía y la salud pública, tal y como plantea un informe coordinado por el profesor Ramón Salaverría para el Ministerio de Ciencia de España (Grupo de Trabajo Multidisciplinar, 20 de febrero de 2021).

En cualquier caso, la respuesta a la desinformación ha de ser una respuesta compleja, como corresponde a un fenómeno poliédrico (Guallar et al., 2020) y multifactorial. Por ello, resulta necesario que esta respuesta proceda de los principales actores informativos y mediáticos, pero también de las redes sociales y de otras empresas tecnológicas (Persily, 2017; Ungría, 2018). Desarrollarla requiere de especialización periodística (Graves y Glaisyer, 2012; Uscinski y Butler, 2013), pero también de nuevas metodologías y herramientas adaptables a las demandas de un sistema informativo cada vez más complejo.

Conocer el perfil del profesional del fact-checking nos parece un paso imprescindible en el proceso de aproximación a una práctica compleja en desarrollo y que está marcando el devenir de los flujos informativos. Si bien la investigación sobre periodismo y nuevos medios ha estudiado ampliamente la adaptación del rol periodístico a las nuevas demandas informativas, el perfil del fact-checker-que, como hemos visto, se distingue del rol periodístico tradicional — ha sido poco analizado hasta el momento y continúa estando poco definido. Son Graves y Cherubini (2016) quienes plantean que el rol del fact-checker va más allá de la práctica periodística al hablar, esencialmente, de tres perfiles de fact-checkers. periodistas, activistas y expertos. Proponemos, a continuación, una evaluación de la situación de desarrollo del factchecking hispanohablante desde la perspectiva de sus profesionales. 
Herrero, E. y Herrera Damas, S. El fact-checker en español alrededor del mundo: Perfil, similitudes y diferencias entre verificadores hispanohablantes

El fact-checking en español, desarrollado en los países de habla hispana, está siguiendo el patrón internacional de crecimiento exponencial. El número de plataformas de habla hispana en activo censadas en el momento de elaborar este trabajo (a fecha de 1 de abril de 2021) es de 46, lo que supone un aumento del $557 \%$ con respecto a las 7 plataformas censadas en 2018 (Palau-Sampio, 2018), un incremento del $142 \%$ con respecto a las 19 censadas en 2019 (Vizoso y VázquezHerrero, 2019), y un crecimiento del $18 \%$ con respecto al censo realizado en el marco de esta investigación en junio de 2020, momento en el que se contabilizaron 39 iniciativas en activo.

La investigación llevada a cabo sobre el fact-checking hispanohablante (PalauSampio, 2018; Vizoso y Vázquez-Herrero, 2019) habla de un ecosistema particular, que hace frente a retos diferentes a los de la verificación anglosajona. Tener en cuenta el contexto informativo, social y político es relevante para el estudio del fact-checking, especialmente en el marco hispano, tal y como plantean Moreno-Gil et al. (2021). Precisamente, este contexto distancia al fact-checking de estos países del modelo estadounidense que tradicionalmente ha sido utilizado para entender este fenómeno.

Consideramos necesario, entonces, atender a distintas variables en el desarrollo de prácticas de verificación, considerando el idioma como un nexo de unión fundamental. En un mundo global, las desinformaciones no conocen fronteras y el idioma común es un punto de vinculación entre los distintos países de habla hispana y sus plataformas de verificación. Entendemos, así, el desarrollo de plataformas como Latam Chequea, que, desde 2020, aúna esfuerzos de iniciativas de distintos países hispanohablantes en la lucha contra la desinformación. Tal y como plantean Graves y Cherubini (2016), el contexto condiciona enormemente las formas de actuar frente a la desinformación. Es por esto que se hace imprescindible un análisis de la implantación del fact-checking en distintos escenarios, superando la óptica anglosajona que, tradicionalmente, viene dominando la investigación sobre verificación de informaciones.

\section{OBJETIVOS}

El objetivo general de este trabajo es examinar la figura del fact-checker en el ámbito hispanohablante. Desde una perspectiva más concreta, los objetivos específicos son:

OE1: esbozar los rasgos del perfil demográfico y profesional del fact-checker en español

OE2: conocer su visión sobre el particular

OE3: examinar la estructura y redes de colaboración que existen entre los diferentes verificadores y

OE4: analizar qué cuestiones tienen en común y cuáles les diferencian a la hora de llevar a cabo su trabajo.

En línea con estos objetivos específicos, planteamos las siguientes 4 preguntas de investigación, habida cuenta del carácter exploratorio de este trabajo: 
Herrero, E. y Herrera Damas, S. El fact-checker en español alrededor del mundo: Perfil, similitudes y diferencias entre verificadores hispanohablantes

PI1: ¿Cuál es el perfil de los profesionales del fact-checking en los países hispanohablantes?

PI2: ¿Qué visión tienen los verificadores hispanohablantes sobre el fenómeno?

PI3: ¿Qué nivel de colaboración existe entre los fact-checkers en español?

PI4: ¿Cuáles son las semejanzas y diferencias entre los profesionales españoles y latinoamericanos en el desarrollo de su trabajo?

\section{METODOLOGÍA}

Para responder a las preguntas de investigación, desarrollamos una metodología mixta que combinó la técnica cuantitativa de la encuesta con la técnica cualitativa de la entrevista en profundidad.

\subsection{La encuesta}

En primer lugar, llevamos a cabo una encuesta a profesionales del fact-checking que trabajan en español. Para ello, elaboramos un censo de iniciativas de verificación en español en activo a partir de la bibliografía existente (Palau Sampio, 2018; Vizoso y Vázquez-Herrero, 2019) y de las bases de datos de Duke Reporters' Lab (2021) y Latam Chequea (2021).

En el momento en que confeccionamos el censo (1 de abril de 2021), el número de plataformas de fact-checking en español en activo era de 46: 41 de ellas se ubicaban en Latinoamérica, 4 en España y 1 -AFP Factual- tenía un carácter multinacional.

Enviamos el cuestionario a todas las plataformas censadas: en los casos en los que fue posible, lo hicimos llegar de manera individualizada a los trabajadores de cada una de las plataformas. Cuando no era posible acceder a sus direcciones electrónicas, enviamos el cuestionario por correo electrónico a cada agencia. Tras 3 correos de recordatorio, obtuvimos respuesta de un total de 52 profesionales, lo que implica una representación de un $47 \%$ sobre el total de plataformas censadas (ver Tabla 1). 
Herrero, E. y Herrera Damas, S. El fact-checker en español alrededor del mundo: Perfil, similitudes y diferencias entre verificadores hispanohablantes

Tabla 1. Desglose de las respuestas recibidas a la encuesta por plataforma y país

\begin{tabular}{|c|c|c|}
\hline Nombre de plataforma & País & $\begin{array}{c}\text { Número de } \\
\text { respuestas } \\
\text { recibidas }\end{array}$ \\
\hline Maldita.es & España & 7 \\
\hline Chequeado & Argentina & 5 \\
\hline Newtral & España & 5 \\
\hline ColombiaCheck & Colombia & 4 \\
\hline Ecuador Chequea & Ecuador & 4 \\
\hline Salud con Lupa & Perú & 4 \\
\hline Fáctica (Agencia Ocote) & Guatemala & 3 \\
\hline Chequea Bolivia & Bolivia & 3 \\
\hline Bolivia Verifica & Bolivia & 2 \\
\hline El Sabueso & México & 2 \\
\hline UyCheck & Uruguay & 2 \\
\hline La Silla Vacía & Colombia & 2 \\
\hline Mala Espina & Chile & 1 \\
\hline PolétikaRD & República Dominicana & 1 \\
\hline Efecto Coyuco & Venezuela & 1 \\
\hline DobleCheck & Perú & 1 \\
\hline Fake News Report & Chile & 1 \\
\hline Chequeando & Chile & 1 \\
\hline De Facto (El Toque) & Cuba & 1 \\
\hline EFE Verifica & España & 1 \\
\hline Fast Check CL & Chile & 1 \\
\hline & & \\
\hline
\end{tabular}

Fuente: Elaboración propia

Para el diseño del cuestionario, utilizamos como guía el conjunto objetivos específicos y preguntas de investigación planteadas. En concreto, diseñamos el cuestionario a partir de los siguientes tres bloques:

- En un primer bloque, para dar respuesta a las preguntas de investigación PI1 y PI4, introdujimos preguntas identificadoras de carácter demográfico y profesional (edad, género, país de nacimiento, formación académica, plataforma de fact-checking en la que trabaja y cargo profesional que ocupa en ella).

- En un segundo bloque, buscando responder a las preguntas de investigación PI3 y PI4, incluimos cuestiones relativas a la actividad profesional de los factcheckers. Así, en este bloque las preguntas giraban en torno a las competencias necesarias para el fact-checking, las técnicas de verificación, redes de colaboración o la configuración de las plantillas.

- En un tercer y último bloque, para responder a la pregunta de investigación PI2, preguntamos a los encuestados por su opinión y visión personal sobre el fenómeno de la verificación. Para ello, interrogamos por su percepción sobre la razón de ser del fact-checking, la necesidad (o no) de establecer redes de colaboración, las dificultades que se encuentran en el desarrollo de su labor y potenciales propuestas de mejora. 
Herrero, E. y Herrera Damas, S. El fact-checker en español alrededor del mundo: Perfil, similitudes y diferencias entre verificadores hispanohablantes

Aunque la mayor parte de las preguntas fueron cerradas -incluyendo la opción "otros" para evitar errores de no cobertura- intercalamos estas preguntas con otras de elección múltiple y en escala, utilizadas para conocer el grado de acuerdo con diferentes afirmaciones. En el diseño del cuestionario tuvimos en cuenta los criterios de claridad, brevedad y neutralidad. Al tratarse de una encuesta auto administrada, tuvimos también en cuenta el orden, la facilidad y la agilidad en la respuesta.

\subsection{Las entrevistas en profundidad}

En paralelo, realizamos 13 entrevistas en profundidad a algunas de las principales voces de la verificación en español ${ }^{3}$, tanto desde una perspectiva profesional como académica. Para seleccionar a los entrevistados profesionales tuvimos en cuenta tanto su vinculación como sus cargos de responsabilidad en las principales plataformas hispanohablantes. En el caso de los entrevistados académicos, consideramos como criterio de selección su experiencia y sus publicaciones académicas vinculadas al factchecking.

Adaptamos la guía a los dos perfiles seleccionados: por un lado, elaboramos un modelo para los entrevistados de corte profesional y otro para los de tipo académico. Teniendo en cuenta la posterior explotación de resultados, organizamos ambos modelos en bloques equivalentes. En concreto:

- Bloque 1: Perfil del entrevistado.

- Bloque 2 (PI2): Cuestiones relativas a la desinformación y su impacto en las sociedades contemporáneas.

- Bloque 3 (PI3 y PI4): Cuestiones relativas al fact-checking, sus dificultades y sus oportunidades en el panorama hispanohablante.

- Bloque 4 (PI1 y PI4): Cuestiones relativas a la necesidad de formación específica en los profesionales de la verificación.

${ }^{3}$ Los 13 entrevistados fueron:

1. Laura Zommer (fundadora y coordinadora de Chequeado, Argentina)

2. Clara Jiménez-Cruz (cofundadora de Maldita.es, España)

3. Desirée García (responsable de EFE Verifica, España)

4. Eliana Álvarez (cofundadora y directora de UyCheck, Uruguay, y coordinadora de la iniciativa Verificado)

5. Ramón Salaverría (en ese momento, vicedecano de Investigación en la Facultad de Comunicación de la Universidad de Navarra, España; auditor de International Fact-Checking Network para las iniciativas hispanohablantes y experto en la materia)

6. Myriam Redondo (profesora y especialista en fact-checking, autora del libro Verificación digital para periodistas)

7. Raúl Magallón Rosa (Universidad Carlos III de Madrid, España; autor de los libros Unfaking News y Desinformación y pandemia: la nueva realidad)

8. Jorge Vázquez Herrero (Universidad de Santiago de Compostela, España; estudioso del fenómeno)

9. María José Ufarte Ruiz (Universidad de Castilla La-Mancha, España; estudiosa del fenómeno),

10. Miguel Carvajal (Universidad Miguel Hernández, España)

11. Leonarda García Jiménez (Universidad de Murcia, España y Colorado State University, EE.UU.)

12. Luis Miguel Pedrero Esteban (Universidad Nebrija, España), y

13. José Luis Rojas Torrijos (Universidad de Sevilla, España). 
Herrero, E. y Herrera Damas, S. El fact-checker en español alrededor del mundo: Perfil, similitudes y diferencias entre verificadores hispanohablantes

- Bloque 5 (PI1): Cuestiones relativas al perfil de los fact-checkers hispanohablantes.

\section{DISCUSIÓN}

A lo largo de este epígrafe, daremos respuesta a las preguntas de investigación que planteamos al inicio de este trabajo a través de los datos recabados mediante la encuesta y las entrevistas en profundidad. La siguiente tabla puede servir de guía (ver Tabla 2).

Tabla 2. Preguntas de investigación, variables generales y fuentes

\begin{tabular}{|c|c|c|c|}
\hline $\begin{array}{l}\text { Pregunta de } \\
\text { investigación }\end{array}$ & Variable general & $\begin{array}{c}\text { Fuente/técnica que } \\
\text { responde a la } \\
\text { pregunta de } \\
\text { investigación }\end{array}$ & $\begin{array}{l}\text { Apartado de la } \\
\text { discusión en el } \\
\text { que se aborda }\end{array}$ \\
\hline $\begin{array}{l}\text { PI1: ¿Cuál es el } \\
\text { perfil de los } \\
\text { profesionales del } \\
\text { fact-checking en } \\
\text { los países } \\
\text { hispanohablantes? }\end{array}$ & $\begin{array}{l}\text { Perfil de los profesionales } \\
\text { en los países } \\
\text { hispanohablantes }\end{array}$ & $\begin{array}{c}\text { Revisión bibliográfica, } \\
\text { encuesta y entrevistas en } \\
\text { profundidad }\end{array}$ & 4.1 \\
\hline $\begin{array}{l}\text { PI2: ¿Qué visión } \\
\text { tienen los } \\
\text { verificadores } \\
\text { hispanohablantes } \\
\text { sobre el } \\
\text { fenómeno? }\end{array}$ & $\begin{array}{l}\text { Percepción de los } \\
\text { profesionales del fact- } \\
\text { checking sobre la } \\
\text { verificación de } \\
\text { informaciones }\end{array}$ & $\begin{array}{c}\text { Encuesta y entrevistas en } \\
\text { profundidad }\end{array}$ & 4.2 \\
\hline $\begin{array}{l}\text { PI3: ¿Qué nivel de } \\
\text { colaboración } \\
\text { existe entre los } \\
\text { fact-checkers en } \\
\text { español? }\end{array}$ & $\begin{array}{c}\text { Iniciativas de colaboración } \\
\text { desarrolladas entre } \\
\text { plataformas de fact- } \\
\text { checking en español }\end{array}$ & $\begin{array}{c}\text { Encuesta y entrevistas en } \\
\text { profundidad }\end{array}$ & 4.3 \\
\hline $\begin{array}{l}\text { PI4: ¿Cuáles son } \\
\text { las semejanzas y } \\
\text { diferencias entre } \\
\text { los profesionales } \\
\text { españoles y } \\
\text { latinoamericanos } \\
\text { en el desarrollo } \\
\text { de su trabajo? }\end{array}$ & $\begin{array}{c}\text { Similitudes y diferencias en } \\
\text { el desempeño de su } \\
\text { trabajo }\end{array}$ & $\begin{array}{c}\text { Encuesta y entrevistas en } \\
\text { profundidad }\end{array}$ & 4.4 y 4.5 \\
\hline
\end{tabular}

Fuente: Elaboración propia

\subsection{El perfil de los verificadores hispanohablantes}

Tomando como referencia la bibliografía previa sobre el tema (Palau Sampio, 2018; Vizoso y Vázquez-Herrero, 2019) y las bases de datos de plataformas de verificación consultadas para la presente investigación, hemos visto que el fact-checking en español es un fenómeno en auge, como también lo es su equivalente a nivel global (Graves, 2016). Así lo demuestran las iniciativas recogidas en nuestro censo y las entrevistas llevadas a cabo con distintos profesionales. La realización de una encuesta 
Herrero, E. y Herrera Damas, S. El fact-checker en español alrededor del mundo: Perfil, similitudes y diferencias entre verificadores hispanohablantes

a verificadores hispanohablantes nos ha permitido conocer cuál es el perfil del profesional de la verificación en español. Exponemos, a continuación, sus rasgos más destacados:

\subsubsection{Perfil demográfico de los verificadores hispanohablantes}

Con respecto a la edad de los fact-checkers, apreciamos que los profesionales son eminentemente jóvenes. La mayoría (un $73,1 \%$ sobre el total de encuestados) tiene menos de 40 años (ver Gráfico 1), siendo mayoritario (42,3\% sobre el total) el grupo de entre 30 y 39 años. En efecto, este perfil joven encaja con los planteamientos de los entrevistados sobre el fact-checking como una nueva salida profesional del periodismo, un fenómeno en auge vinculado a las redes sociales, el periodismo digital y las nuevas tecnologías.

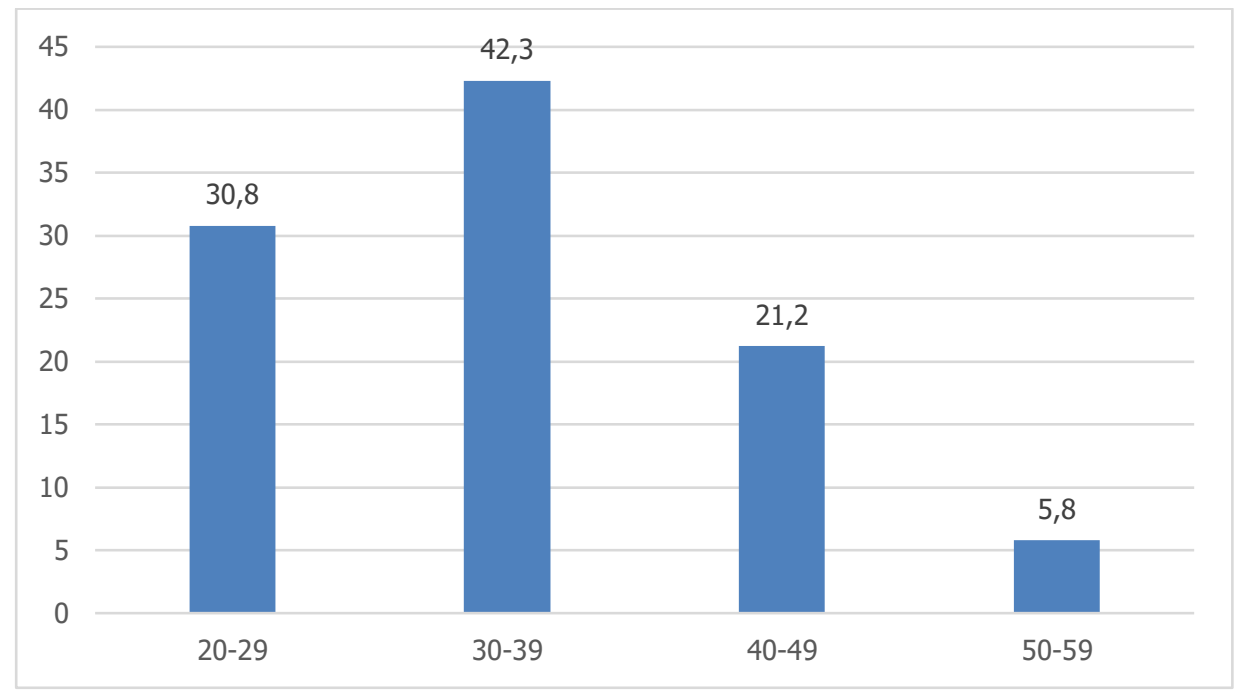

Gráfico 1: Edad de los fact-checkers hispanohablantes (sobre el \% total de la muestra)

Fuente: Elaboración propia

En cuanto al género, vemos que hay una distribución equitativa: el $53,8 \%$ de los encuestados son hombres y el $46,2 \%$ son mujeres. Con respecto al país de nacimiento, apreciamos que el fact-checking es un fenómeno bastante extendido en los países de habla hispana; es decir, que su desarrollo no se limita a unos pocos países: profesionales de 19 de los 21 países hispanohablantes (el 90,5\%) están representados en la muestra.

Nos hemos interesado también por la formación y especialización de los profesionales de la verificación. Entendiendo el fact-checking como un fenómeno periodístico (Graves, 2016), consideramos relevante conocer la relación de quienes se dedican profesionalmente a la verificación con los programas académicos de formación periodística y comunicativa. 
De esta forma, en lo que refiere a la formación académica, podemos ver que el del fact-checker es un perfil cualificado en el contexto hispano: todos los encuestados indican ser titulados superiores. Atendiendo, particularmente, a la especialización en verificación, vemos que un $48,1 \%$ del total de los encuestados manifiesta haber recibido, además, algún tipo de formación específica en este sentido.

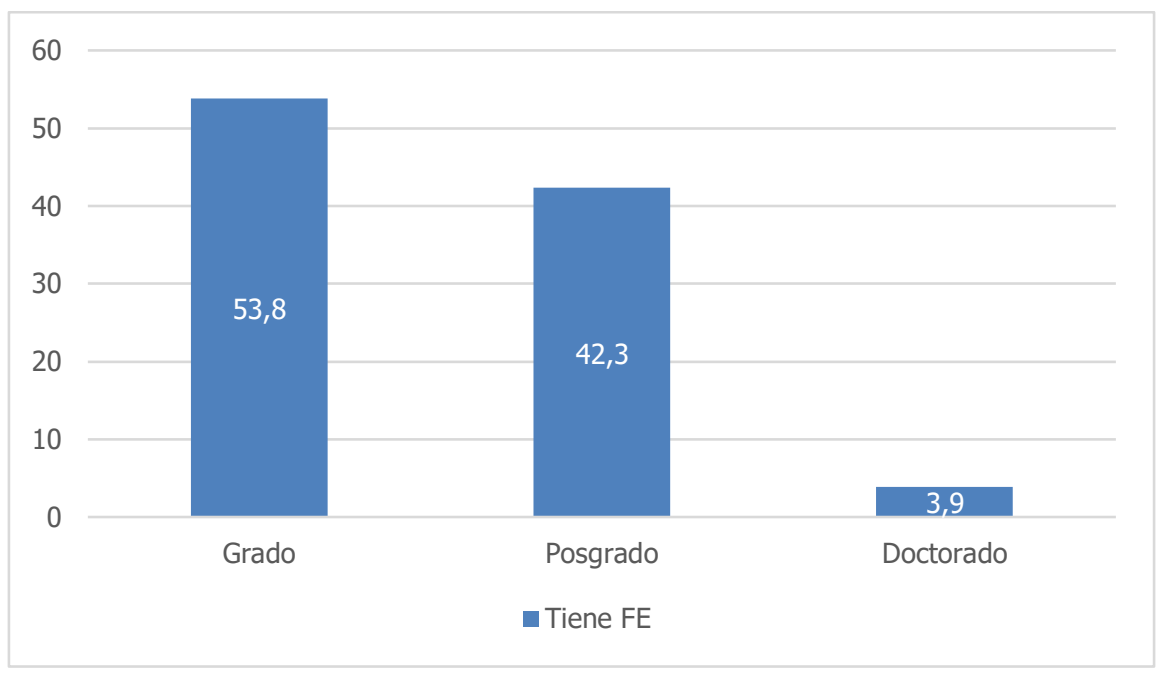

Gráfico 2: Formación académica de los fact-checkers (sobre el \% total de la muestra)

Fuente: Elaboración propia

Por otra parte, si atendemos a la especialización temática, apreciamos que un $40,4 \%$ de los encuestados manifiesta no estar especializado en un tema concreto, mientras que un $21,2 \%$ indica estarlo en más de un asunto. Este elevado porcentaje nos muestra, en efecto, el carácter interdisciplinar y multitemático del fact-checking, pese a su indispensable vinculación con la comunicación política, el periodismo político y la verificación del discurso público (Uscinski y Butler, 2013; Luengo y García-Marín, 2020). Precisamente, un $23,1 \%$ de los encuestados indica estar especializado en estas temáticas. Otras especializaciones minoritarias son las relativas a cuestiones de género, economía, migraciones, salud y medio ambiente o comunicación. 
Herrero, E. y Herrera Damas, S. El fact-checker en español alrededor del mundo: Perfil, similitudes y diferencias entre verificadores hispanohablantes

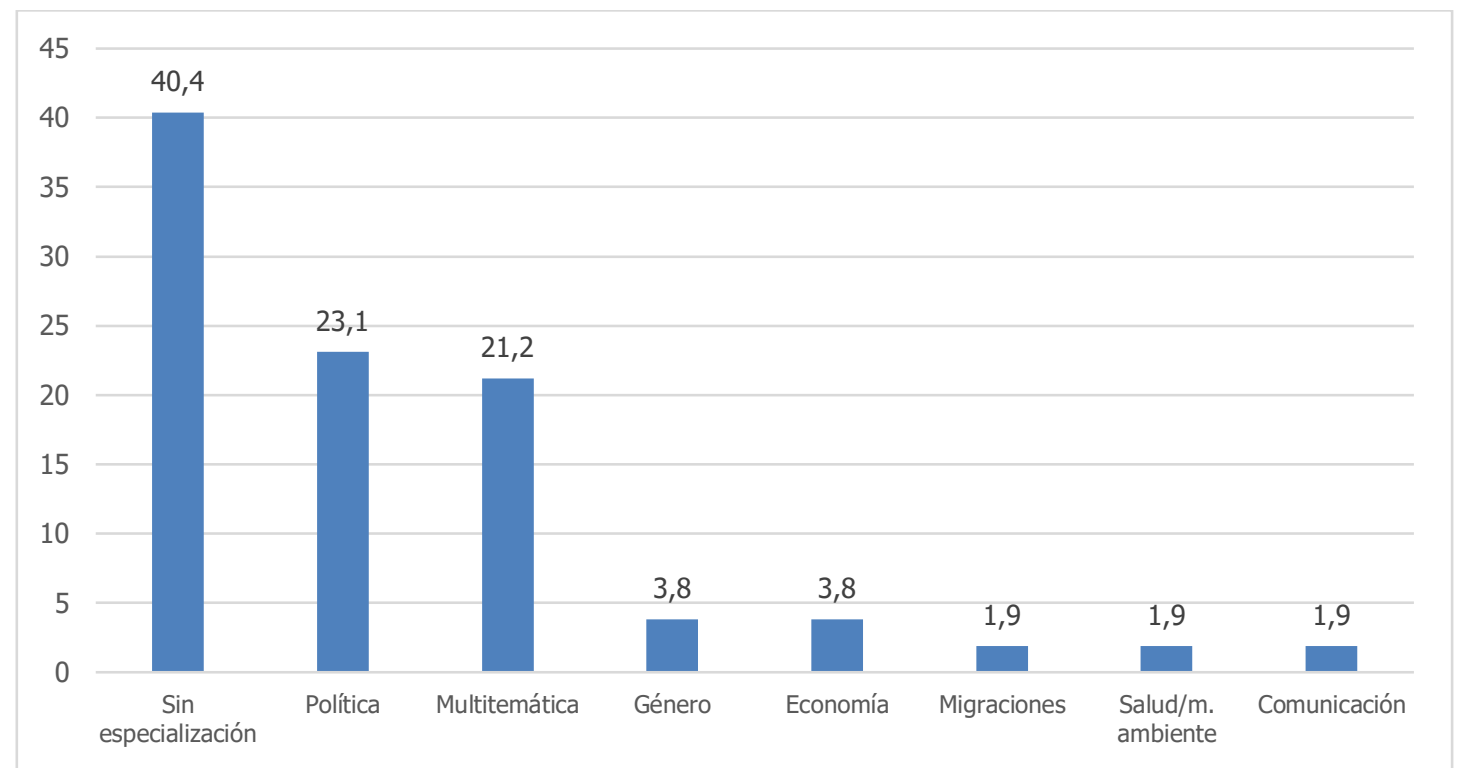

Gráfico 3: Tipo de especialización temática de los fact-checkers (sobre el \% total de la muestra)

Fuente: Elaboración propia

\subsubsection{Perfil profesional de los verificadores hispanohablantes}

Los resultados de la encuesta revelan que un $30,8 \%$ son directores o fundadores de las plataformas; un $21,2 \%$, editores; un $11,5 \%$, jefes de sección y un $36,5 \%$, redactores.

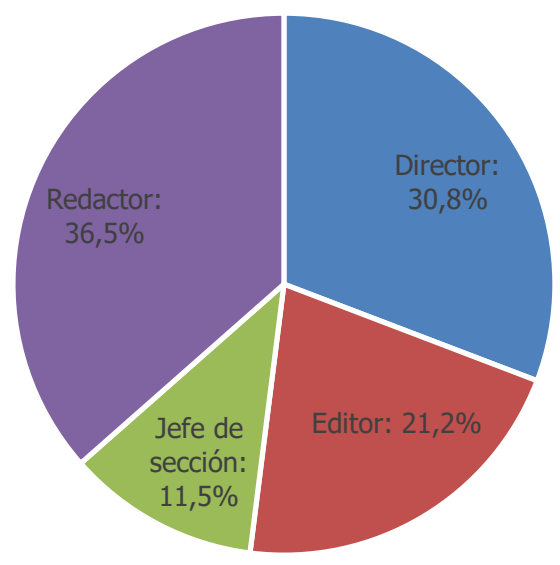

Gráfico 4: Categoría profesional de los encuestados (sobre el \% total de la muestra) Fuente: Elaboración propia

La alta tasa de directores con respecto a otras categorías profesionales se podría explicar por el reducido tamaño de las plantillas que tiene la mayoría de fact-checkers en español en Latinoamérica, mencionada como un elemento característico del fact- 
checking latinoamericano por la fundadora de Chequeado, Laura Zommer. En este sentido, vemos que un $44 \%$ de los fact-checkers hispanohablantes trabaja en plataformas de menos de 6 trabajadores. Un 25\% lo hace en plataformas medianas (entre 7 y 15 trabajadores); un 19\% en plataformas medianas-grandes (entre 15 y 30 ) y apenas un $12 \%$ en plataformas grandes (más de 30 ).

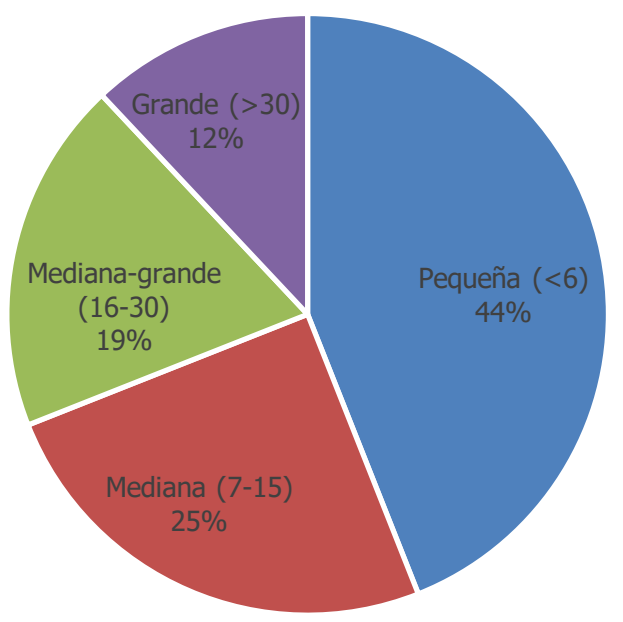

Gráfico 5: Tamaño de las plataformas de fact-checking hispanohablantes según su número de trabajadores

Fuente: Elaboración propia

\subsection{Visión sobre el fact-checking de los verificadores hispanohablantes}

Con respecto a la visión particular del fact-checking que tienen los profesionales de habla hispana, hemos analizado dos tipos de cuestiones: en primer lugar, las relativas a las competencias necesarias para verificar; $y$, en segundo lugar, las relativas a la percepción de hasta qué punto el fact-checking está vinculado al actual contexto social y mediático.

\subsubsection{Competencias necesarias para la verificación}

Para un $94,2 \%$ de los encuestados, la formación periodística resulta una competencia capital. En menor medida, se cita también el dominio del big data (esencial para un $71,2 \%$ de la muestra) y el manejo de redes sociales (necesarias para el $44,2 \%$ de los encuestados). Los datos para el conjunto de la muestra se pueden apreciar en el siguiente gráfico: 
Herrero, E. y Herrera Damas, S. El fact-checker en español alrededor del mundo: Perfil, similitudes y diferencias entre verificadores hispanohablantes

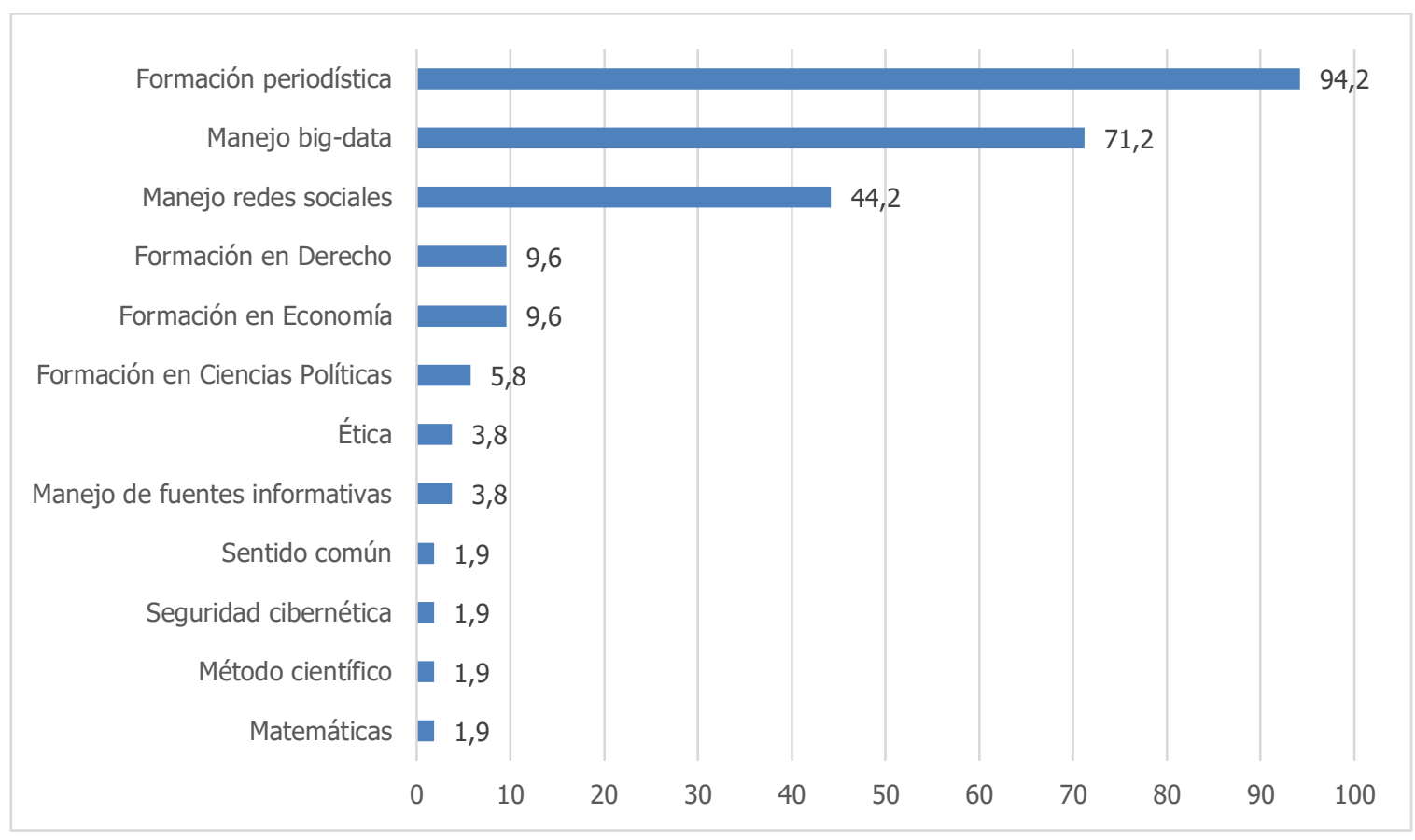

Gráfico 6: Percepción de competencias necesarias para el fact-checking (sobre el \% total de los encuestados)

Fuente: Elaboración propia

En este sentido, y como ya planteaban Kovach y Rosenstiel (2007) o Amorós García (2019), también algunas de las profesionales entrevistadas apuntan a la vinculación intrínseca del fact-checking con el periodismo: "Es indudable que los fact-checkers somos periodistas, pero, sobre todo, que los periodistas son fact-checkers" (JiménezCruz, 2020); "[La verificación] está ahora muy vinculada al periodismo, pero lo estará cada vez más a la inteligencia artificial" (Redondo, 2020).

También expertos académicos en fact-checking apuntan a esta vinculación con el periodismo: "Es una tarea que se ha hecho siempre en la historia del periodismo y es natural al trabajo periodístico" (Carvajal, 2020); "Es algo de toda la vida, que se llama verificación y que es parte sustancial de lo que debe entenderse como una de las obligaciones de cualquier medio periodístico" (Rojas-Torrijos, 2020).

\subsubsection{Vinculación del fact-checking con el actual contexto social y mediático}

Preguntados por la vinculación entre la verificación y el actual ecosistema social y mediático, los encuestados se muestran mayoritariamente de acuerdo con la necesidad de esta para hacer frente a las problemáticas desencadenadas en los nuevos procesos comunicativos mediáticos. Los encuestados consideran que el fact-checking tiene efectos positivos sobre la calidad y la veracidad del discurso político y sobre los flujos informativos. También declaran, de manera mayoritaria, entender el fact-checking como una herramienta útil en momentos de crisis. 
Herrero, E. y Herrera Damas, S. El fact-checker en español alrededor del mundo: Perfil, similitudes y diferencias entre verificadores hispanohablantes

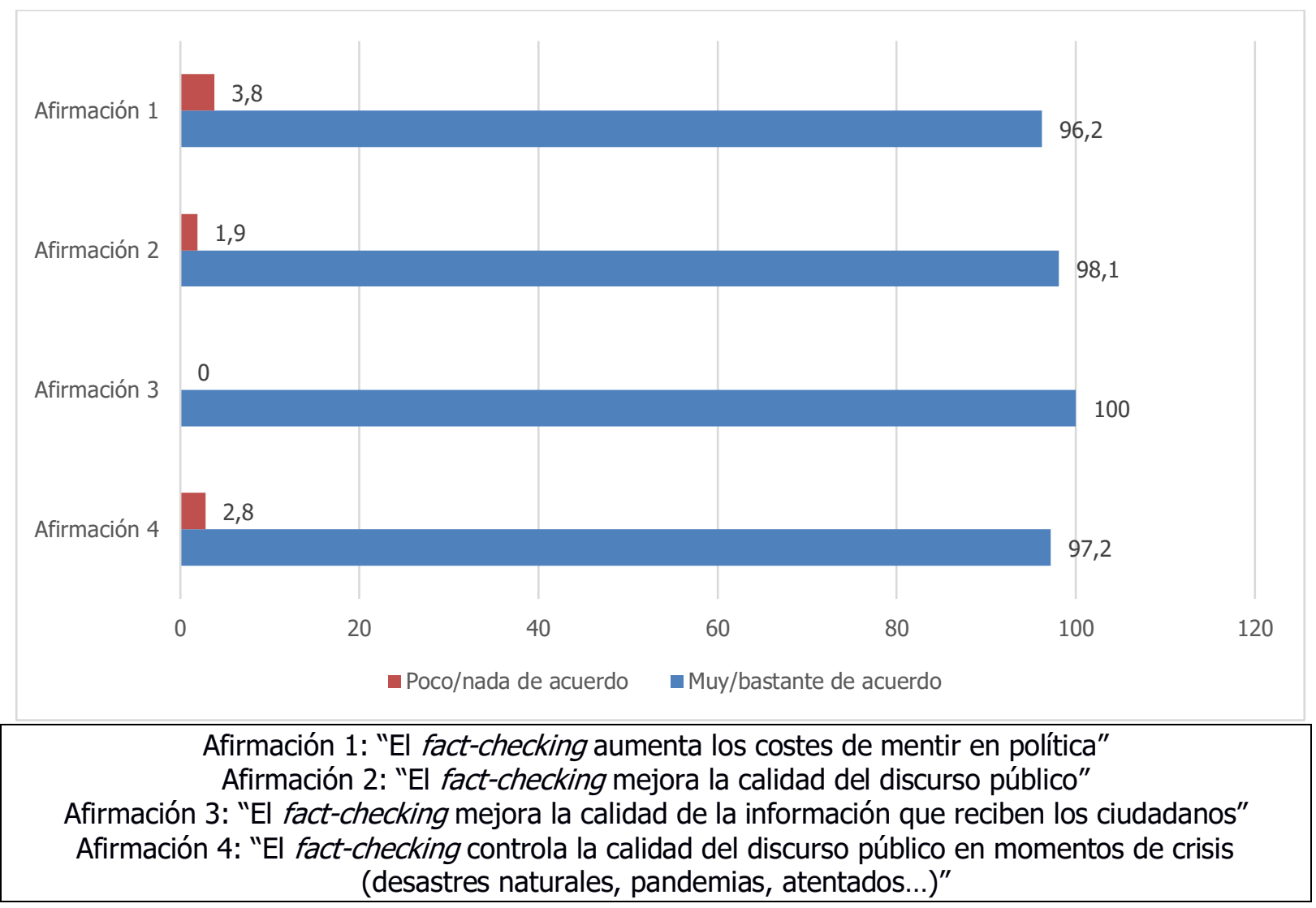

Gráfico 7: Porcentaje de acuerdo con afirmaciones acerca de la relevancia del factchecking en la sociedad actual (en \% sobre el total de la muestra)

Fuente: Elaboración propia

Esta idea de que el fact-checking es una herramienta clave en el control de la información política, del discurso público y, en general, de la información que llega a las audiencias, es compartida por los expertos entrevistados. Así, Clara Jiménez-Cruz (cofundadora de Maldita.es) apunta a la relevancia del fact-checking como herramienta al servicio de los ciudadanos:

La realidad es que, con las nuevas formas de consumo de información, a través de dispositivo móvil, internet... se ha facilitado la transmisión de información, pero también de desinformación y por lo tanto es necesario reaprender a hacer ese consumo. Mientras vamos reaprendiendo cómo tenemos que consumir e identificar lo que es información de lo que no lo es en esas nuevas plataformas, está bien que tengamos alguien que nos eche una mano para darnos información sobre lo que es real y sobre lo que es falso, y, a partir de ahí, cada uno toma la decisión que toma sobre lo que quiere (Jiménez-Cruz, 2020).

Esta idea es compartida por Eliana Álvarez (cofundadora de UyCheck) que apunta al fact-checking como una respuesta periodística a un problema de sobreinformación generado por las nuevas tecnologías:

Estamos en un momento donde el fact-checking es necesario porque circula muchísima más información y a una velocidad mucho más grande de la que lo que hacía antes. Si bien la desinformación y los políticos mintiendo, o diciendo 
Herrero, E. y Herrera Damas, S. El fact-checker en español alrededor del mundo: Perfil, similitudes y diferencias entre verificadores hispanohablantes

cosas falsas, existieron desde siempre, ahora la velocidad y el alcance es mucho más grande, pero a la vez también es relativo. Es especialmente importante ahora porque tenemos los canales y la información disponible para hacer esa verificación, antes tal vez no era tan sencillo (Álvarez, 2020).

Expertos académicos como Ramón Salaverría (Universidad de Navarra) apuntan a que el fact-checking certifica, en cambio, algunas de las deficiencias del periodismo actual:

Yo pienso que el fact-checking en realidad es una evidencia de que no estamos haciendo el periodismo todo lo bien que deberíamos estar haciéndolo, porque si el periodismo que realizan los medios profesionales tuviera la excelencia profesional que es deseable y las plataformas de Internet tuvieran los filtros para eliminar la diseminación indiscriminada de contenidos ficticios, no serían necesarias las plataformas de verificación (Salaverría, 2020).

En esta misma línea, Amazeen había señalado que el fact-checking "podría ser un síntoma del declive de las instituciones periodísticas de referencia" (2017, p. 5).

Por otra parte, nos hemos interesado en conocer la visión que los fact-checkers hispanohablantes tienen sobre la vinculación entre la verificación y las redes sociales, una cuestión que, como hemos visto (Cazalens et al., 2018; Graves, 2018), ha sido estudiada por la bibliografía académica y que resulta fundamental a la hora de entender las potenciales automatizaciones de los procesos verificadores.

En este sentido, destaca la percepción mayoritaria que tienen los encuestados $\left(98^{\prime} 1 \%\right)$ sobre la relevancia de los usuarios como vía de acceso a los bulos que circulan por redes sociales cerradas. Como planteaban los entrevistados, el contexto mediático hispanohablante destaca por un empleo de redes sociales cerradas (encriptadas), como WhatsApp, que no es tan común en el mundo anglosajón. Así lo expresaba la cofundadora de Maldita.es, Clara Jiménez-Cruz (2020): "La desinformación, en países hispanohablantes ... está en WhatsApp". Los encuestados también se posicionan a favor de que tanto las redes sociales abiertas (Twitter, Facebook...) como las cerradas (WhatsApp, Telegram...) colaboren con las plataformas de verificación. 
Herrero, E. y Herrera Damas, S. El fact-checker en español alrededor del mundo: Perfil, similitudes y diferencias entre verificadores hispanohablantes

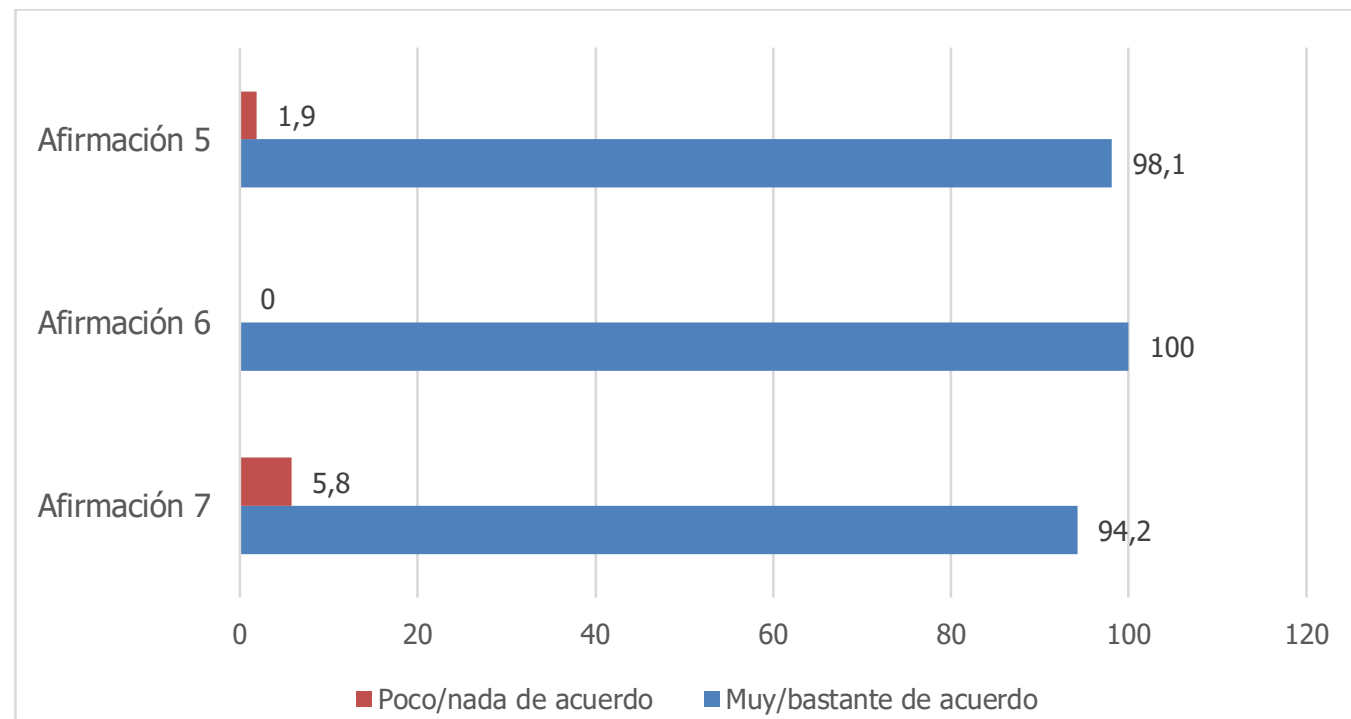

Afirmación 5: "Los usuarios son una importante fuente de acceso a los bulos, sobre todo los que circulan por redes sociales cerradas"

Afirmación 6: "Es importante que las redes sociales abiertas (Twitter, Facebook...) colaboren con los fact-checkers para filtrar el contenido que circula en ellas o para avisar a los usuarios de la existencia de desinformaciones"

Afirmación 7: "Es importante que las redes sociales cerradas (WhatsApp, Telegram...) colaboren con los fact-checkers para filtrar el contenido que circula en ellas o para avisar a los usuarios de la existencia de desinformaciones"

Gráfico 8: Porcentaje de acuerdo con afirmaciones acerca de la vinculación del factchecking y las redes sociales (en \% sobre el total de la muestra)

Fuente: Elaboración propia

La relevancia de la colaboración de los usuarios a la hora de controlar la desinformación se menciona de manera recurrente. La propia Jiménez-Cruz comentaba en un seminario para Reuters en mayo de 2020, que "si quieres luchar una batalla, necesitas un ejército", de manera que "cuando empezamos a desarrollar esta estrategia les dimos un nombre, empezamos a llamarlos 'malditas' y 'malditos' ... y nos dimos cuenta de que empezaban a vincularse con nosotros" ${ }^{\prime \prime}$. Destacan así las iniciativas de distintas plataformas hispanohablantes para establecer vías de contacto directo con sus usuarios, como el ChatBot de Maldita.es (Maldita.es, junio 2020) o la posibilidad de hacerse "chequeadista" de Chequeado (Chequeado, 2021).

\subsection{Nivel de colaboración entre fact-checkers hispanohablantes}

En lo que refiere a la colaboración entre plataformas, la cofundadora de Chequeado, Laura Zommer, comenta que el nacimiento de la International Fact-Checking Network (IFCN) en 2014 está muy vinculado a esta necesidad:

\footnotetext{
${ }^{4}$ Literalmente, "If you want to fight a battle, you need an army ... when we started doing this kind of strategy, we needed to give them a name. We called them 'malditas' and 'malditos' and at a certain point we realized that they had started engaging with us".

Cfr. https://www.youtube.com/watch?v=ZHBdpmb9kMI\&feature=youtu.be (min. 14.09).
} 
Herrero, E. y Herrera Damas, S. El fact-checker en español alrededor del mundo: Perfil, similitudes y diferencias entre verificadores hispanohablantes

La IFCN ... surgió por iniciativa de los propios chequeadores que empezamos a juntarnos informalmente en 2014 ... surgió un poco como una necesidad de intercambio de buenas prácticas y también de errores. Muchas organizaciones teníamos los mismos desafíos, en distintos países y con algunos contextos diferenciados, pero también con algunos puntos muy comunes, porque en todos los países hay líderes que no son accurate ${ }^{3}$ o que no dicen siempre la verdad (Zommer, 2020).

En efecto, parece haber cierto consenso en que la desinformación tiende a seguir un camino común en países con contextos sociopolíticos tan paralelos como los latinoamericanos. Así lo plantea también la responsable de UyCheck, Eliana Álvarez, quien llega a apuntar que, en muchos casos, en América Latina, "fueron los propios sitios de fact-checking que ya existían los que fueron promoviendo que en otros países también surgieran [iniciativas]" (Álvarez, 2020). Álvarez afirma también que, "aunque hay cosas que son locales, en realidad, mucha de la desinformación que circula es la misma en todos los países" (Álvarez, 2020). Algo con lo que coincide Laura Zommer (Chequeado) que indica que su plataforma, Chequeado, fundó en 2020 la red Latam Chequea: "Es una red aún más informal que IFCN porque no tiene una construcción legal. Es, claramente, una red de prácticas de organizaciones que hacen chequeo ... en un contexto particular, en los países de Latinoamérica" (Zommer, 2020). La propia Zommer menciona que el marco sociopolítico, informativo y comunicacional de América Latina cuenta con unas dificultades comunes que no aparecen en otros países:

En general, en los países de Latinoamérica, hay algunas dificultades extra que no están necesariamente en los países de, entre comillas, "el primer mundo". No todos los países tienen normas de acceso a la información que se implementan correctamente, o, si las tienen, tienen gaps muy grandes entre lo que dice la norma y la implementación. No todos los países tienen buena calidad de sus datos ... Hay algunos casos, además, donde hay directamente, en ciertos países, persecuciones a cualquiera que se anime a decirle a un presidente, 0 ministro, o funcionario que no está diciendo la verdad a los ciudadanos... Entonces, generamos este espacio para intercambiar algunos desafíos propios de la región (Zommer, 2020).

A los países de Latinoamérica, comenta la directora de Chequeado, "no solo nos une la lengua, sino también algunas de las particularidades de nuestros estados, como más debilidad institucional de nuestras democracias, que nos parecía interesante poder articular para fortalecernos" (Zommer, 2020). En general, añade Eliana Álvarez (UyCheck), "conocer en qué están trabajando los otros aliviana mucho el proceso de trabajo local, porque es posible que ese bulo o esa información que circula en Uruguay ya haya estado en Argentina, Chile y Ecuador"; y comenta que "cuando la información es clave, no conoce fronteras, por lo menos en América Latina" (Álvarez, 2020).

Si la colaboración es esencial en el contexto latinoamericano, también lo es para los fact-checkers españoles. Las redes colaborativas latinoamericanas, como Latam

${ }^{3}$ Precisos.

Revista de Comunicación de la SEECI. 2021, no 54, 49-77 
Herrero, E. y Herrera Damas, S. El fact-checker en español alrededor del mundo: Perfil, similitudes y diferencias entre verificadores hispanohablantes

Chequea, también cuentan con la participación de plataformas españolas, como Newtral o Maldita.es (Latam Chequea, 2021), una cooperación que se hace especialmente necesaria en contextos de desinformación global, como la pandemia por coronavirus, tal y como mencionaba Zommer.

Desde la perspectiva española, la cofundadora de Maldita.es, Clara Jiménez-Cruz, indica que el trabajo colaborativo resulta esencial para los nuevos medios periodísticos, en general. Los servicios de fact-checking, como nuevos medios, también se benefician de la cooperación:

Dentro del mundo del fact-checking, la cooperación se asume y se entiende, sobre todo porque es como se entiende un poco el funcionamiento del periodismo moderno. Con lo diezmadas que están las redacciones, y cuando cada vez los medios especializados son más pequeños ... en ese ambiente en el que los medios son especializados y pequeños, la colaboración es necesaria para poder llegar a todo: hacer trabajos grandes e investigar a gran escala (Jiménez-Cruz, 2020).

Los resultados, en efecto, revelan que un $69,2 \%$ de los encuestados afirma haber colaborado con otras plataformas. De estos, un $100 \%$ lo ha hecho en alguna ocasión con plataformas hispanohablantes tal como se puede apreciar en el siguiente gráfico:

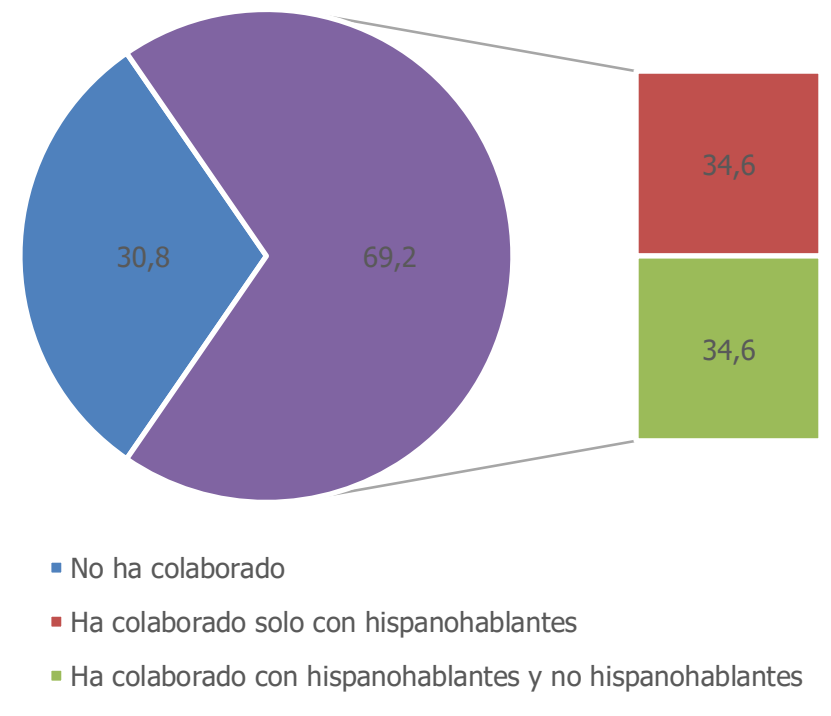

Gráfico 9: Colaboración con otras plataformas según su idioma (en \% sobre el total de la muestra)

Fuente: Elaboración propia

En cuanto a los elementos que favorecen la colaboración interplataformas, los encuestados apuntan especialmente a trabajar con técnicas de verificación similares, a compartir una misma percepción sobre la razón de ser del fact-checking, o pertenecer a las mismas organizaciones internacionales. Para un $48,1 \%$ de los encuestados, compartir el idioma resulta relevante a la hora de colaborar. Otras cuestiones como la proximidad geográfica, en cambio, apenas son destacadas. 
Herrero, E. y Herrera Damas, S. El fact-checker en español alrededor del mundo: Perfil, similitudes y diferencias entre verificadores hispanohablantes

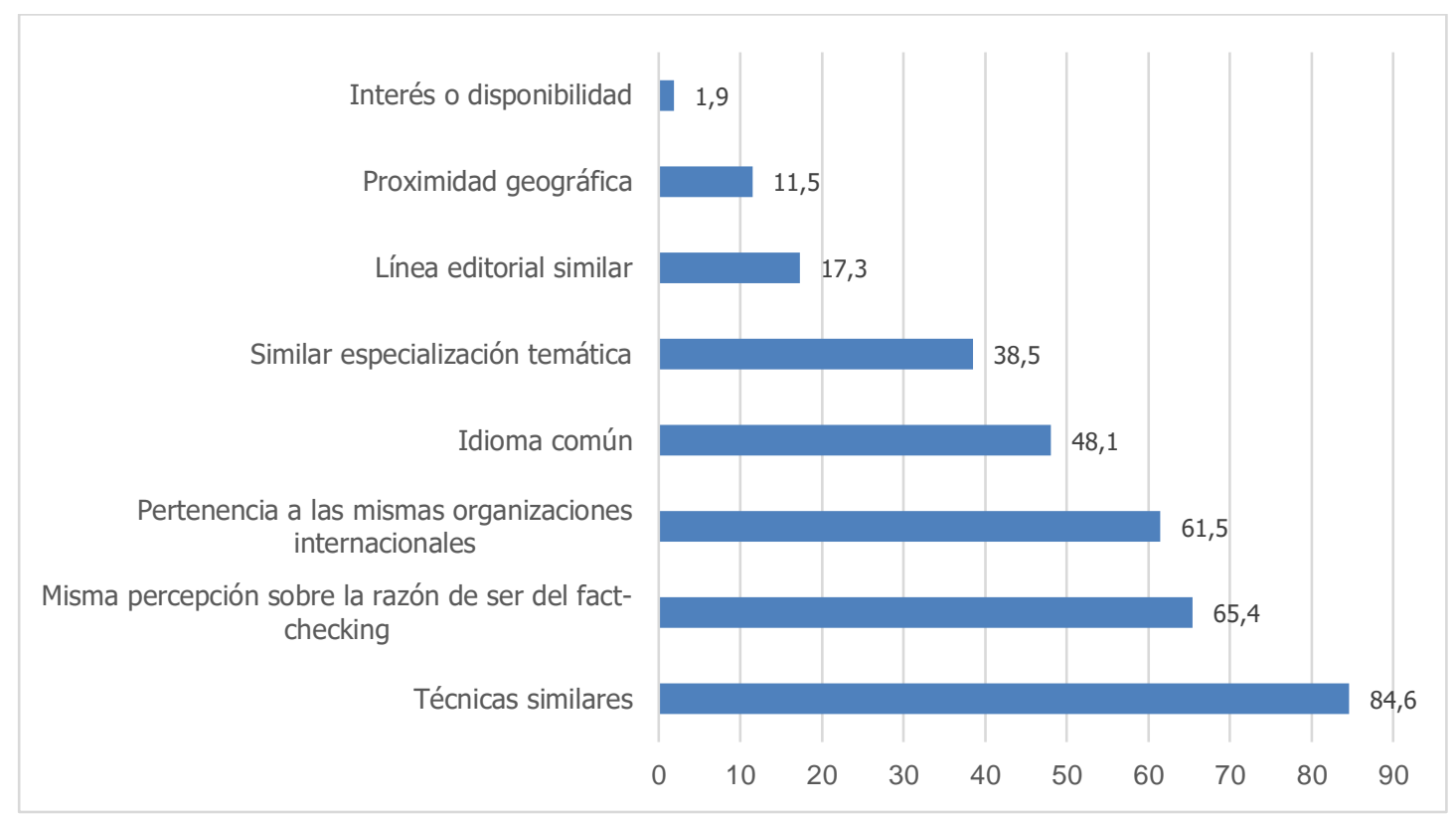

Gráfico 10: Elementos que favorecen la colaboración entre plataformas (en \% sobre el total de la muestra)

Fuente: Elaboración propia

\subsection{Similitudes entre los verificadores hispanohablantes}

Con el objetivo de conocer en profundidad el perfil del fact-checker hispano, y teniendo en cuenta las reflexiones previas sobre los distintos contextos sociopolíticos -español y latinoamericano- que dividen al fact-checking en español (Moreno Gil et al., 2021), nos hemos propuesto comparar a los verificadores hispanohablantes españoles y latinoamericanos.

Encontramos similitudes, fundamentalmente, en torno a dos ejes: el perfil demográfico y la visión sobre la razón de ser de la verificación. En general, en ambos contextos, vemos que el del fact-checker es un perfil joven y cualificado, una tendencia que encaja también con los rasgos de la verificación a nivel internacional (Graves y Cherubini, 2016). Los verificadores hispanohablantes también comparten una visión del fact-checking como movimiento periodístico global que responde, a su vez, a una necesidad informativa y mediática internacional (Graves, 2016). Este eje de similitudes nos permite entender, de manera particular, el fenómeno de la verificación hispanohablante como un objeto de estudio relevante que, pese a las diferencias lógicas entre dos espacios sociales y políticos distintos -España y Latinoamérica-, mantiene un núcleo en común que se materializa, como hemos visto, en la posibilidad de colaboración y en el desarrollo de iniciativas de cooperación internacional como Latam Chequea, entre otras. 
Herrero, E. y Herrera Damas, S. El fact-checker en español alrededor del mundo: Perfil, similitudes y diferencias entre verificadores hispanohablantes

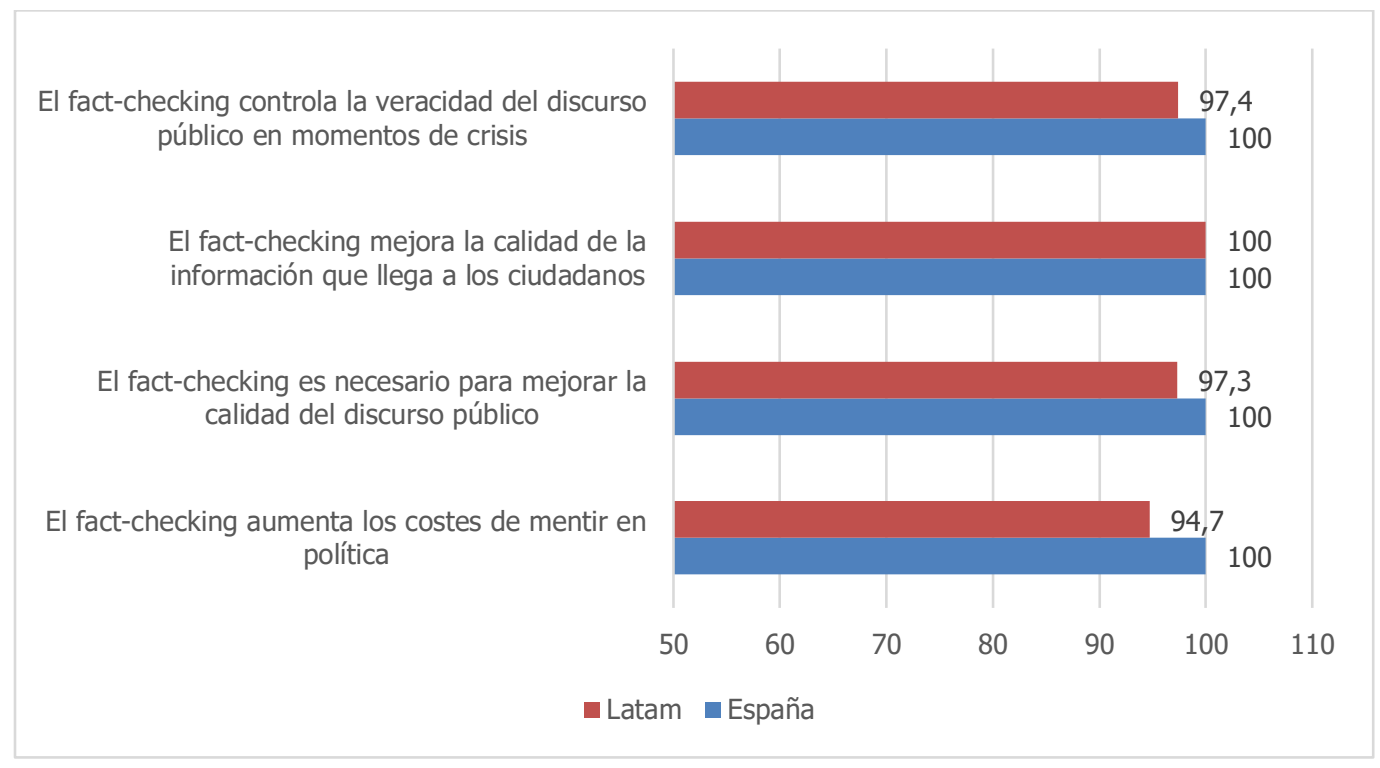

Gráfico 11: Porcentaje de acuerdo con afirmaciones sobre la razón de ser del factchecking (\% de encuestados que manifiestan estar "muy" o "bastante de acuerdo" sobre el total de la muestra)

Fuente: Elaboración propia

\subsection{Diferencias entre los verificadores hispanohablantes}

De la misma manera, nos hemos interesado en conocer cuáles son las principales diferencias entre verificadores españoles y verificadores latinoamericanos.

Pese a que, como hemos podido ver, el perfil demográfico de los fact-checkers es similar, encontramos una diferencia fundamental en cuanto al tamaño de las plataformas en las que trabajan. Las plataformas latinoamericanas son más numerosas y más pequeñas, mientras que en España más de la mitad de las plataformas están consolidadas y tienen un número elevado de trabajadores. Así lo reflejan el censo de iniciativas que hemos elaborado, los resultados de la encuesta y las entrevistas en profundidad. Esta diferencia, que hemos esbozado anteriormente, queda reflejada en los resultados de la encuesta de la siguiente manera: apenas un 5,3\% de los factcheckers latinoamericanos declara trabajar en plataformas grandes (30 o más trabajadores), frente a un $28,6 \%$ de los encuestados españoles. Los datos relativos al conjunto de los encuestados quedan expresados en el siguiente gráfico: 
Herrero, E. y Herrera Damas, S. El fact-checker en español alrededor del mundo: Perfil, similitudes y diferencias entre verificadores hispanohablantes

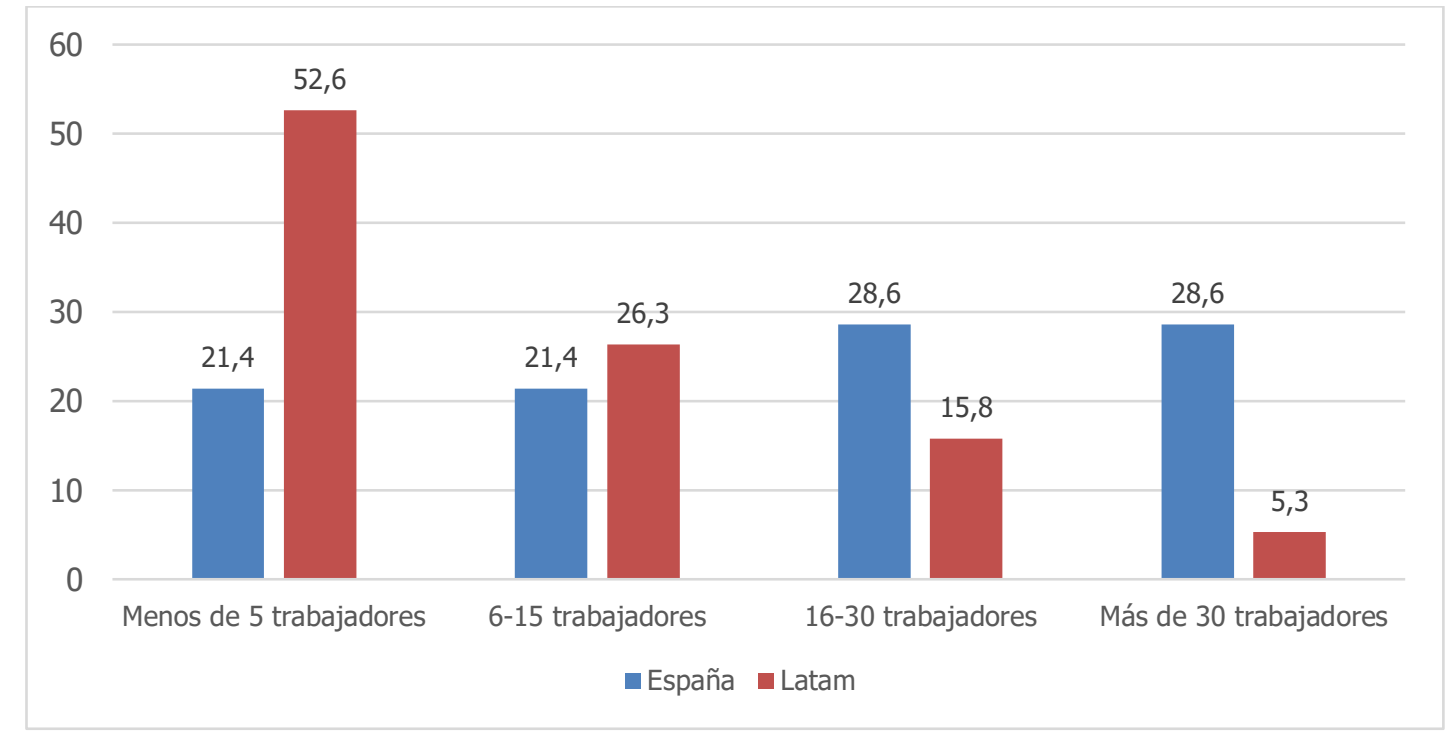

Gráfico 12: Tamaño de las plataformas de fact-checking según no de trabajadores (en \% sobre el total de la muestra)

Fuente: Elaboración propia

Como hemos visto, esta diferencia es un elemento característico del fact-checking latinoamericano. En una entrevista para esta investigación, Laura Zommer (directora de Chequeado, Argentina) explicaba que, en el contexto latinoamericano, la mayor parte de plataformas son muy pequeñas: "En América Latina se da que la mayoría de las organizaciones son organizaciones de menos de cinco o seis personas, las que se dedican exclusivamente a chequeo" (Zommer, 2020). En efecto, vemos que dos de las cuatro plataformas españolas censadas para esta investigación (Newtral y Maldita.es) se cuentan entre las más grandes en número de trabajadores del panorama hispano. En este grupo de plataformas con mayor número de trabajadores estaría también la argentina Chequeado, que supone una excepción entre las plataformas de Sudamérica.

Por otra parte, encontramos una diferencia fundamental en la percepción de qué requisitos son necesarios para que se dé una mayor colaboración entre las distintas iniciativas. Los resultados de la encuesta y las entrevistas en profundidad nos remiten a dos contextos de colaboración organizados en torno a ejes distintos. Si bien la colaboración entre España y Latinoamérica se hace fundamental, también es cierto que la organización y pertenencia a redes de colaboración está mucho más consolidada en el contexto latinoamericano que en el español (un $21,1 \%$ de los encuestados latinoamericanos consideran estas redes fundamentales para la colaboración, frente a un $7,7 \%$ de los españoles). En España se privilegia, entre otras cosas, compartir unas mismas técnicas y metodologías de trabajo (un $84,6 \%$ de los españoles plantea la similitud de técnicas y metodologías como esencial para colaborar, frente a un 55,3\% de los latinoamericanos). A este respecto, Clara Jiménez-Cruz apunta:

Cuando estamos en una situación normal [no en pandemia], mucha de la desinformación es exclusiva de España. Luego hay una parte que tiene que ver con Europa, en general ... es desinformación que compartimos porque vemos los mismos tipos [de bulos] que nuestros socios europeos ... Habitualmente, la 
Herrero, E. y Herrera Damas, S. El fact-checker en español alrededor del mundo: Perfil, similitudes y diferencias entre verificadores hispanohablantes

desinformación política [española] no tiene nada que ver con la latinoamericana (Jiménez-Cruz, 2020).

En este sentido, la colaboración de fact-checkers españoles se hace en dos direcciones: por un lado, en el marco europeo, con el que se comparten cuestiones políticas y geográficas; por otro, aunque en menor medida, en el marco hispanohablante, con el que se comparten cuestiones de similitud lingüística. Así lo plantea la cofundadora de Maldita.es:

Con el coronavirus, estamos viendo muchas cosas con Latinoamérica que tienen mucho sentido y, de hecho, estamos en un proyecto de colaboración llamado Latam Chequea con verificadores hispanohablantes y brasileños en Latinoamérica. $Y$ estamos en un proyecto europeo que estamos coordinando FullFact y Maldita, con los países más afectados [por el coronavirus] en Europa ahora mismo (Jiménez-Cruz, 2020).

En efecto, la colaboración de España con el fact-checking europeo en el marco de la pandemia de coronavirus se ha materializado en iniciativas como el informe realizado por AFP, CorrectITV, Pagella Politica, FullFact y Maldita.es sobre la circulación de bulos sobre salud en Europa 5 .

Desde la perspectiva latinoamericana, Laura Zommer (Chequeado) indica que la colaboración con plataformas españolas en Latam Chequea, en el marco de la COVID19, se entiende porque "el virus llegó antes a Europa que a América Latina. Para América Latina era bien relevante que muchas de las desinformaciones que fueron llegando a nuestros países ya habían pasado por Europa, o por Asia, o por EE.UU." (Zommer, 2020). La colaboración con España, en este caso, resultaba de interés porque:

Más allá de la red internacional de chequeadores ... no todas nuestras audiencias leen inglés, entonces nos parecía importante que también generáramos algo para tener nuestra respuesta más veloz cada vez que aparece una desinformación. Sabemos que el tiempo, sobre todo en desinformación viral, es un factor crítico: cuanto antes reaccionemos a la desinformación, menos chances hay de que llegue más lejos (Zommer, 2020).

Desirée García (EFE Verifica) explica que el idioma es un factor clave: "Muchos contenidos, por el hecho de circular en la misma lengua, se viralizan muy rápido tanto en Latinoamérica como en España" y, pese a ello, coincide en que la desinformación española está especialmente vinculada a Europa:

También estamos sometidos [en España] a otro tipo de desinformación que, a lo mejor, no sufren tanto en Latinoamérica, que es la que procede sobre todo de actores que la propia Unión Europea ha señalado como desestabilizadores (García-Pruñonosa, 2020).

${ }^{5} \mathrm{Cfr}$. https://covidinfodemiceurope.com/?lang=es

Revista de Comunicación de la SEECI. 2021, n 54, 49-77 
Herrero, E. y Herrera Damas, S. El fact-checker en español alrededor del mundo: Perfil, similitudes y diferencias entre verificadores hispanohablantes

\section{CONCLUSIONES}

Tomando como referencia las preguntas de investigación planteadas al inicio, a lo largo de este trabajo hemos esbozado el perfil de los profesionales del fact-checking en los países hispanohablantes. De esta forma, hemos visto que se trata de un perfil considerado esencialmente periodístico y vinculado de manera estrecha a las lógicas periodísticas.

Los verificadores hispanohablantes son principalmente jóvenes y cualificados, si bien apenas un $50 \%$ de ellos ha recibido formación específica en verificación de informaciones. Como fenómeno en crecimiento, la formación académica en factchecking está aún en desarrollo y en ocasiones son las propias plataformas las que están llevando a cabo iniciativas formativas para futuros profesionales del área, una tendencia ya consolidada en el mundo anglosajón (Graves, 2016). En España, Newtral y Maldita.es colaboran con distintas universidades: Newtral ofrece un máster en verificación digital, fact-checking y periodismo de datos junto a la Universidad CEU San Pablo; Maldita.es, por su parte, ofrece a partir del curso 2021/2022 un máster en investigación periodística, nuevas narrativas, datos, fact-checking y transparencia junto a la Universidad Rey Juan Carlos.

Al tratarse de un fenómeno relativamente reciente, no está especialmente consolidado en el contexto hispanohablante donde la mayoría de plataformas son pequeñas. Sin embargo, agencias como Chequeado, Maldita.es y Newtral, con plantillas grandes, se están convirtiendo en modelos de referencia a nivel internacional y están, a la vez, contribuyendo al desarrollo de la verificación en el panorama hispano mediante iniciativas de cooperación como Latam Chequea.

Con respecto a la colaboración, encontramos que, precisamente por el carácter pequeño y volátil de muchas de las plataformas latinoamericanas, la cooperación interplataformas se constituye como una necesidad. En España, no obstante, la colaboración se da en dos direcciones: a nivel sociopolítico y geográfico, las plataformas españolas tienden a colaborar más con otras agencias europeas; sin embargo, el idioma compartido con Latinoamérica convierte la colaboración hispana en una realidad cada vez más relevante, especialmente en contextos de crisis global, como la crisis sanitaria de la COVID-19.

Las similitudes entre los fact-checkers españoles y latinoamericanos son evidentes: el perfil demográfico de los trabajadores, las percepciones sobre el fact-checking y sobre la colaboración son elementos en común que convierten al fact-checking en español en un fenómeno relevante para entender el desarrollo de la verificación más allá de los límites anglosajones. No obstante, encontramos algunas diferencias que responden a los diferentes contextos sociopolíticos de los que hablamos, especialmente diferencias en torno a los elementos necesarios para la colaboración, la necesidad de plataformas de cooperación internacional o el tamaño y consolidación de las agencias de fact-checking.

Revista de Comunicación de la SEECI. 2021, no 54, 49-77 
Herrero, E. y Herrera Damas, S. El fact-checker en español alrededor del mundo: Perfil, similitudes y diferencias entre verificadores hispanohablantes

En cualquier caso, exponemos aquí una aproximación a este fenómeno, escasamente abordado hasta ahora desde la perspectiva de los implicados. Entendemos que, en adelante, será necesario y relevante entender las dinámicas de colaboración, interrelación y cooperación que se dan entre los países hispanos, con independencia de sus contextos particulares.

\section{LIMITACIONES Y FUTURAS LÍNEAS DE INVESTIGACIÓN}

A pesar de ser un trabajo descriptivo de un área poco explorada hasta el momento, la presente investigación está limitada por el reducido tamaño de la muestra y por la imposibilidad de delimitar un universo de fact-checkers hispanohablantes. Las conclusiones alcanzadas sirven para esbozar un terreno aún por explorar como es la verificación de informaciones en español. Además, el rápido crecimiento y desarrollo que está experimentando el fact-checking hispano dificulta la posibilidad de obtener una foto fija del fenómeno.

Futuras líneas de trabajo podrían ampliar este boceto abordando con mayor profundidad aspectos considerados relevantes por los propios implicados tales como las redes colaboración entre plataformas, las diferencias y similitudes entre profesionales a nivel nacional o la idiosincrasia del fact-checking hispano con respecto a otros más estudiados, como el anglosajón.

\section{REFERENCIAS}

Adair, B. (2019). El fact checking es lo más importante del periodismo en la era digital. elmundo.es.

https://www.elmundo.es/television/2019/07/25/5d38750afc6c833d428b4625.htm

Algan, Y., Guriev, S., Papaioannou, E., \& Passari, E. (2017). The European trust crisis and the rise of populism. Brookings Papers on Economic Activity, 309-400. https://doi.org/10.1353/eca.2017.0015

Amazeen, M. A. (2017). Journalistic interventions. The structural factors affecting the global emergence of fact-checking. Journalism, 21(1), 95-111. https://doi.org/10.1177\%2F1464884917730217

Amorós, M. (2018). Fake News: la verdad de las noticias falsas. Barcelona: Plataforma Editorial.

Brennen, J. S., Simon, F., Howard, P., \& Kleis Nielsen, R. (2020). Types, sources and claims of COVID-19 misinformation. Reuters Institute. https://bit.ly/3bRTA1H

Cazalens, S., Lamarre, P., Leblay, J., Manolescu, I., \& Tannier, X. (2018). A content management perspective on fact-checking. En WWW'18 Companion: The 2018 Web Conference Companion, 23-27 abril, Lyon: Francia. https://doi.org/10.1145/3184558.3188727 
Herrero, E. y Herrera Damas, S. El fact-checker en español alrededor del mundo: Perfil, similitudes y diferencias entre verificadores hispanohablantes

Chequeado (2021). Apoyá a Chequeado. Chequeado. https://bit.ly/3hPfID7

Ciampaglia, G., Shiralkar, P., Rocha, L., Bollen, J., Menczer, F., \& Flammini, A. (2015). Computational Fact-Checking from Knowledge Networks. Plos One, 10(10). https://doi.org/10.1371/journal.pone.0141938

Coleman, A. (2020). Hundreds dead' because of Covid-19 misinformation. BBC. https://www.bbc.com/news/world-53755067

Del Fresno, M. (2018). Posverdad y desinformación: guía para perplejos. El País. https://elpais.com/elpais/2018/03/16/opinion/1521221740 078721.html

Ginsberg, L., \& Gori, P. (2021). Report on a survey for fact checkers on COVID-19 vaccines and disinformation. European Digital Media Observatory. https://cadmus.eui.eu/handle/1814/70917

Graves, L. (2016). Boundaries not drawn: Mapping the institutional roots of the global fact-checking movement. Journalism Studies, 19(5), 613-631. https://doi.org/10.1080/1461670X.2016.1196602

Graves, L. (2018). Understanding the promise and limits of automated fact-checking. Reuters Institute for the Study of Journalism. https://ora.ox.ac.uk/objects/uuid:f321ff43-05f0-4430-b978-f5f517b73b9b

Graves, L., \& Cherubini, F. (2016). The Rise of Fact-Checking Sites in Europe. Reuters Institute for the Study of Journalism. https://ora.ox.ac.uk/objects/uuid:d55ef650e351-4526-b942-6c9e00129ad7

Graves, L., \& Glaisyer, T. (2012). The fact-checking universe in Spring 2012. Media Policy Initiative.

Grupo de Trabajo Multidisciplinar (2020). Informe del GTM - Grupo de Trabajo Multidisciplinar. Entender y combatir la desinformación sobre ciencia y salud. https://bit.ly/3fd75Lw

Hassan, N., Arslan, F., Li, C., \& Tremayne, M. (2017). Toward automated factchecking: Detecting check-worthy factual claims by Claimbuster. KDD '17: Proceedings of the 23rd ACM SIGKDD International Conference on Knowledge Discovery and Data Mining (pp. 1803-1812). https://doi.org/10.1145/3097983.3098131

International Fact-Checking Network (2016). State of the fact-checkers. https://bit.ly/3xdd2hj

Kovach, B., \& Rosenstiel, T. (2001). The elements of journalism. What Newspeople Should Know and the Public Should Expect. The Crown Publishing Group. 
Herrero, E. y Herrera Damas, S. El fact-checker en español alrededor del mundo: Perfil, similitudes y diferencias entre verificadores hispanohablantes

Latam Chequea (2021). Latam Chequea Coronavirus. https://chequeado.com/latamcoronavirus/

Luengo, M., \& García-Marín, D. (2020). The performance of truth: politicians, factchecking journalism, and the struggle to tackle COVID-19 misinformation. American Journal of Cultural Sociology, 8(3), 405-427. https://doi.org/10.1057/s41290-020$\underline{00115-\mathrm{w}}$

Maldita.es (2020). Maldita.es lanza el primer chatbot de WhatsApp en español para verificar bulos automáticamente. Maldita.es. https://maldita.es/recibe-losdesmentidos-de-maldito-bulo-en-whatsapp

Moreno-Gil, V., Ramon, X., \& Rodríguez-Martínez, R. (2021). Fact-Checking Interventions as Counteroffensives to Disinformation Growth: Standards, Values, and Practices in Latin America and Spain. Media and Communication, 9(1), 251263. http://dx.doi.org/10.17645/mac.v9i1.3443

OMS (2020). Call for action. Managing the infodemic. World Health Organization. https://bit.ly/2Tb2dxP

Palau Sampio, D. (2018). Fact-checking and scrutiny of power: Supervision of public discourses in new media platforms from Latin America. Communication and Society, 31(3), 347-365. https://doi.org/10.15581/003.31.3.347-365

Persily, N. (2017). The 2016 U.S. Election: Can Democracy Survive the Internet? Journal of Democracy, 28(2), 63-76. https://doi.org/10.1353/jod.2017.0025

Poynter (2021). The International Fact-Checking Network. https://www.poynter.org/ifcn/

Rose, J. (2017). Brexit, Trump and Post Truth Politics. Public Integrity, 19(6), 555-558. https://doi.org/10.1080/10999922.2017.1285540

Scientific American (2020). Covid misinformation is killing people. Disponible en: https://www.scientificamerican.com/article/covid-misinformation-is-killing-people1/

Ungría, C. (2018). Twitter y Facebook podrían hacer mucho más para luchar contra los bulos. TreceBits. https://bit.ly/3wA5yVL

Uscinski, J., \& Butler, R. (2013). The Epistemology of Fact Checking. Critical Review, 25, 162-80. https://doi.org/10.1080/08913811.2013.843872

Vizoso, A., López-García, X. y Pereira-Fariña, X. (2018). Habilidades tecnológicas en el perfil del fact-checker para la verificación de información en la sociedad red. Estudos em Comunicaçao, 271), 105-126. https://doi.org/10.20287/ec.n27.v1.a07 
Herrero, E. y Herrera Damas, S. El fact-checker en español alrededor del mundo: Perfil, similitudes y diferencias entre verificadores hispanohablantes

Vizoso, Á. y Vázquez-Herrero, J. (2019). Plataformas de fact-checking en español. Características, organización y método. Communication and Society, 32(1), 127144. https://doi.org/10.15581/003.32.1.127-144

\section{AUTOR/ES:}

\section{Esperanza Herrero}

Graduada en Periodismo (2019) e Información y Documentación (2019) con Premio Extraordinario de Fin de Grado, y Máster en Investigación Aplicada a Medios de Comunicación (2020). Actualmente es investigadora predoctoral FPU/MECD en el Departamento de Comunicación de la Facultad de Comunicación y Documentación, en la Universidad de Murcia (España). Sus líneas de investigación están centradas en la teoría de la comunicación, los estudios de género e identidad, la verificación de informaciones y el fact-checking.

mariaesperanza.herrero@um.es

Orcid ID: https://orcid.org/0000-0001-5926-2142

Google Scholar: https://scholar.google.es/citations?user=FLseN94AAAAJ\&hl=es

ResearchGate: https://www.researchgate.net/profile/Esperanza-Herrero

\section{Susana Herrera Damas}

Licenciada en Comunicación Audiovisual (1998) y en Sociología (2004) y Doctora en Comunicación Audiovisual (2002). Premio Extraordinario de Doctorado, es autora de 5 libros y de más 80 artículos publicados en revistas de prestigio. Hasta diciembre de 2016 cuenta con 3 sexenios de investigación reconocidos por la CNEAI y desde noviembre de 2020 está acreditada como Catedrática por la ANECA. Ha sido profesora visitante en las Universidades de Ottawa, Texas y Missouri. En la actualidad, es profesora titular de Periodismo en el Departamento de Comunicación de la Universidad Carlos III de Madrid (España) donde dirige el grupo de investigación Innovation on Digital Media y el programa de doctorado en Investigación en Medios de Comunicación. dherrera@hum.uc3m.es

Orcid ID: https://orcid.org/0000-0002-1755-1621

Google Scholar: https://scholar.google.es/citations?user=p4u4vfAAAAAJ\&hl=es

ResearchGate: https://www.researchgate.net/profile/Susana-Herrera-Damas https://researchportal.uc3m.es/display/inv37900 